\title{
Optimal Real-time Pricing Algorithm Based on Utility Maximization for Smart Grid
}

\author{
Pedram Samadi, Amir-Hamed Mohsenian-Rad, Robert Schober, Vincent W.S. Wong, and Juri Jatskevich \\ Department of Electrical and Computer Engineering \\ The University of British Columbia, Vancouver, Canada \\ E-mail:\{psamadi, hamed, rschober, vincentw, jurij\}@ece.ubc.ca
}

\begin{abstract}
In this paper, we consider a smart power infrastructure, where several subscribers share a common energy source. Each subscriber is equipped with an energy consumption controller (ECC) unit as part of its smart meter. Each smart meter is connected to not only the power grid but also a communication infrastructure such as a local area network. This allows two-way communication among smart meters. Considering the importance of energy pricing as an essential tool to develop efficient demand side management strategies, we propose a novel real-time pricing algorithm for the future smart grid. We focus on the interactions between the smart meters and the energy provider through the exchange of control messages which contain subscribers' energy consumption and the real-time price information. First, we analytically model the subscribers' preferences and their energy consumption patterns in form of carefully selected utility functions based on concepts from microeconomics. Second, we propose a distributed algorithm which automatically manages the interactions among the ECC units at the smart meters and the energy provider. The algorithm finds the optimal energy consumption levels for each subscriber to maximize the aggregate utility of all subscribers in the system in a fair and efficient fashion. Finally, we show that the energy provider can encourage some desirable consumption patterns among the subscribers by means of the proposed real-time pricing interactions. Simulation results confirm that the proposed distributed algorithm can potentially benefit both subscribers and the energy provider.
\end{abstract}

\section{INTRODUCTION}

Electricity is currently provided through an infrastructure consisting of utility companies, power plants, and transmission lines which serve millions of customers. For example, the electric power grid in the United States includes more than 3,100 electric utilities operating more than 10,000 power plants, and there are about 157,000 miles of high voltage electric transmission lines which bring energy to more than 131 million customers [1]. The dependency of almost all parts of industry and different aspects of our life on electrical energy makes this massive infrastructure a strategic entity.

Given the increased expectations of customers, both in quality and quantity [1], the limited energy resources, and the lengthy and expensive process of exploiting new resources, the reliability of the grid has been put in danger and there is a need to develop new methods to increase the grid efficiency. Currently, the electricity consumption is not efficient in most buildings (e.g., due to poor thermal isolation). This results in the waste of a large amount of natural resources, since most of the electricity consumption occurs in buildings [2]. In addition, the arising of new types of demand such as plug-in hybrid electric vehicles (PHEVs), which can potentially double the average household load, have further increased the need to develop new methods for demand side management (DSM).

There is a wide range of DSM techniques such as voluntary load management programs [3]-[5] and direct load control [6]. However, smart pricing is known as one of the most common tools that can encourage users to consume wisely and more efficiently. Given the recent increases in the price of energy, the users are more willing to improve the insulation conditions of their buildings or try to shift the energy consumption schedule of their high-load household appliances to off-peak hours. DSM has been considered since the early 1980s [7][11]. DSM can be used as a tool for load shaping, where the electricity demand is being re-distributed over a certain period of time (e.g., time-of-day, day-of-week). Broad categories of load shaping objectives include peak clipping, load shifting, valley filling, strategic conservation, and flexible load shaping [7]. For example, peak clipping includes direct load control of the utilities on customers' appliances to reduce the peak load.

Several pricing schemes have already been proposed in the smart grid literature. In general, flat pricing, peak load pricing, and adaptive pricing are among the most popular approaches to pricing which have been practiced extensively [12]-[15]. Flat pricing refers to those methods where the utility company announces a fixed price for all periods. In peak load pricing, the intended cycle is divided into several periods and a distinct price value for each period is announced at the beginning of the operation [14]. On the other hand, in adaptive pricing, instead of announcing a pre-determined price for each period of operation at the beginning of the day, the exact price value for each period is calculated in real-time and is announced only at the beginning of each operation period. Clearly, in this method, the realization of random events and the reaction of users with respect to the previous prices will influence the price in the upcoming operation periods [12].

Based on a report of the U.S. Department of Energy [16], smart grid is an electricity delivery system enhanced with communication facilities and information technologies to enable more efficient and reliable grid operation with an improved customer service and a cleaner environment. By exploiting the two-way communication capabilities of smart meters it becomes possible to replace the current power system with a more intelligent infrastructure [17]. From this and given the importance of demand side management, in this paper, 
we focus on the real-time interactions among subscribers and the energy provider and introduce a novel real-time pricing algorithm for the future smart grid. The contributions of this paper can be summarized as follows:

- We propose a real-time pricing algorithm for DSM programs to encourage desired energy consumption behaviors among users and to keep the total consumption level below the power generation capacity.

- In our system model, the subscribers and the energy provider automatically interact with each other through a limited number of message exchanges and by running a distributed algorithm to find the optimal energy consumption level for each subscriber, the optimal price values to be advertised by the energy provider, and also the optimal generating capacity for the energy provider.

- We model the subscriber's preferences and their energy consumption patterns in form of carefully selected utility functions based on concepts from microeconomics.

- We formulate the real-time pricing as an optimization problem to maximize the aggregate utility of all subscribers in the system while minimizing the imposed energy cost to the energy provider. Moreover, we include constraints to limit the total energy consumption level of all users to the total electricity generation capacity of the system offered by the energy provider.

- We prove the existence and the uniqueness of the optimal solution for the formulated optimization problem.

- Simulation results confirm that both subscribers and the energy provider will benefit from the proposed algorithm.

This paper is organized as follows. The system model is presented in Section II. In Section III, we formulate our design as a convex optimization problem and propose a distributed pricing algorithm. Simulation results are given in Section IV, and conclusions are drawn in Section V.

\section{SySTEM MODEL}

Consider a smart power system consisting of a single energy provider, several load subscribers or users, and a regulatory authority. For each user, we assume that there is an energy consumption controller (ECC) unit which is embedded in the user's smart meter. The role of the ECC is to control the user's power consumption, and to coordinate each user with other users and also with the energy provider. All ECC units are connected to each other and to the energy provider through a communication infrastructure such as a local area network.

The intended time cycle for the operation of the users is divided into $K$ time slots, where $K \triangleq|\mathcal{K}|$, and $\mathcal{K}$ is the set of all time slots. This division can be based on the behavior of the users and their power demand pattern: peak load time slots, valley load time slots, and normal load time slots. Also, let $\mathcal{N}$ denote the set of all users, where $N \triangleq|\mathcal{N}|$. For each user $i \in \mathcal{N}$, let $x_{i}^{k}$ denote the amount of power consumed by user $i$ in time slot $k$. For each subscriber $i \in \mathcal{N}$ and each time slot $k \in \mathcal{K}$, we define the power consumption interval $I_{i}^{k}$ as

$$
I_{i}^{k} \triangleq\left[m_{i}^{k}, M_{i}^{k}\right]
$$

and the consumed power $x_{i}^{k}$ has to satisfy $m_{i}^{k} \leq x_{i}^{k} \leq M_{i}^{k}$. Here, $m_{i}^{k}$ and $M_{i}^{k}$ denote the minimum and the maximum power consumption of user $i$, respectively. The minimum power consumption level may represent the load from appliances such as refrigerator which always need to be on during the day. The maximum power consumption level may also represent the total power consumption level of household appliances assuming that all appliances are on.

The regulatory authority ensures that the energy provider will provide the minimum capacity to cover the minimum power requirements of all users $L_{k}^{\text {min }}$ in each time slot.

$$
L_{k}^{\text {min }} \triangleq \sum_{i \in \mathcal{N}} m_{i}^{k}, \quad \forall k \in \mathcal{K} .
$$

The generation capacity in each time slot $k \in \mathcal{K}$ is denoted by $L_{k}$, which may differ among time slots. We also define $L_{k}^{\max }$ as the maximum generating capacity in each time slot $k \in \mathcal{K}$.

\section{A. User Preference and Utility Function}

Each individual subscriber in a power system is an entity which can behave independently. The energy demand of each subscriber may vary based on different parameters. For example, we can take into account the time of day, climate conditions, and also the price of electricity. The energy demand also depends on the type of the users. For example, household users may have different responses to the same price than industrial users. The different response of different users to various price scenarios can be modeled analytically by adopting the concept of utility function from microeconomics [18]. In fact, we can model the behavior of different users through their different choices of utility functions [4]. For all users, we represent the corresponding utility function as $U(x, \omega)$, where $x$ is the power consumption level of the user and $\omega$ is a parameter which may vary among users and also at different times of the day. More formally, for each user, the utility function represents the level of satisfaction obtained by the user as a function of its power consumption. We assume that the utility functions fulfill the following properties:

1) Property I: Utility functions are non-decreasing. That is, users are always interested to consume more power if possible until they reach their maximum consumption level. Mathematically, this implies that we have

$$
\frac{\partial U(x, \omega)}{\partial x} \geq 0
$$

For notational convenience we define

$$
V(x, \omega) \triangleq \frac{\partial U(x, \omega)}{\partial x},
$$

as the marginal benefit [3], [4].

2) Property II: The marginal benefit of customers is a nonincreasing function and we have

$$
\frac{\partial V(x, \omega)}{\partial x} \leq 0
$$

In other words, the utility functions are concave and the level of satisfaction for users can gradually get saturated. While the class of utility functions that fulfill (3) and (5) is very large, it is convenient to have a linear marginal benefit [3], [4]. 
3) Property III: We have to be able to rank the customers based on their utilities. In our formulation, we assume, for a fixed consumption level $x$, a larger $\omega$ implies a larger $U(x, \omega)$, which can be expressed as

$$
\frac{\partial U(x, \omega)}{\partial \omega}>0 .
$$

4) Property IV: We assume the general expectation that no power consumption brings no benefit, so we have

$$
U(0, \omega)=0, \quad \forall \omega>0 .
$$

Various choices of utility functions are widely used in the communications and networking literature [19]. However, recent reports indicate that the behavior of power users can also be accurately modeled by certain utility functions [3]. In this paper, we consider quadratic utility functions corresponding to linear decreasing marginal benefit [5]:

$$
U(x, \omega)=\left\{\begin{array}{llr}
\omega x-\frac{\alpha}{2} x^{2} & \text { if } & 0 \leq x \leq \frac{\omega}{\alpha}, \\
\frac{\omega}{\alpha} & \text { if } & x \geq \frac{\omega}{\alpha},
\end{array}\right.
$$

where $\alpha$ is a pre-determined parameter. Sample utility functions from this class are shown in Fig. 1.

A subscriber that consumes $x \mathrm{~kW}$ electricity during a designated number of hours at a rate of $P$ dollars per $\mathrm{kWh}$ is charged $P x$ dollars per hour. Hence, the welfare of each user can simply be represented as

$$
W(x, \omega)=U(x, \omega)-P x,
$$

where $W(x, \omega)$ is the user's welfare function, $U(x, \omega)$ is the utility function of the user, $P x$ is the cost imposed by the energy provider to the user, and $x$ is the user's power consumption. For each announced price value $P$, each user tries to adjust its power consumption $x$ to maximize its own welfare, and this can be achieved by setting the derivative of (9) equal zero which means that at the optimal consumption level, the marginal benefit of the user would be equal to the announced price. For example, different power consumption responses of a user with a decreasing linear marginal benefit to two different announced prices are depicted in Fig. 2.

\section{B. Energy Cost Model}

We consider a cost function $C_{k}\left(L_{k}\right)$ indicating the cost of providing $L_{k}$ units of energy offered by the energy provider in each time slot $k \in \mathcal{K}$. We make the following assumptions:

Assumption 1: The cost functions are increasing in the offered energy capacity. That is, for each $k \in \mathcal{K}$, we have

$$
C_{k}\left(\hat{L}_{k}\right) \leq C_{k}\left(\tilde{L}_{k}\right), \quad \forall \hat{L}_{k} \leq \tilde{L}_{k} .
$$

Assumption 2: The cost functions are strictly convex. For each $k \in \mathcal{K}$, any $0 \leq \theta \leq 1$, and $\hat{L}_{k}, \tilde{L}_{k} \geq 0$, we have [20]

$$
C_{k}\left(\theta \hat{L}_{k}+(1-\theta) \tilde{L}_{k}\right) \leq \theta C_{k}\left(\hat{L}_{k}\right)+(1-\theta) C_{k}\left(\tilde{L}_{k}\right) .
$$

Piece-wise linear functions and quadratic functions are two example cost functions that satisfy Assumption 1 and Assumption 2. In this paper, we consider quadratic cost functions [10]:

$$
C_{k}\left(L_{k}\right)=a_{k} L_{k}^{2}+b_{k} L_{k}+c_{k},
$$

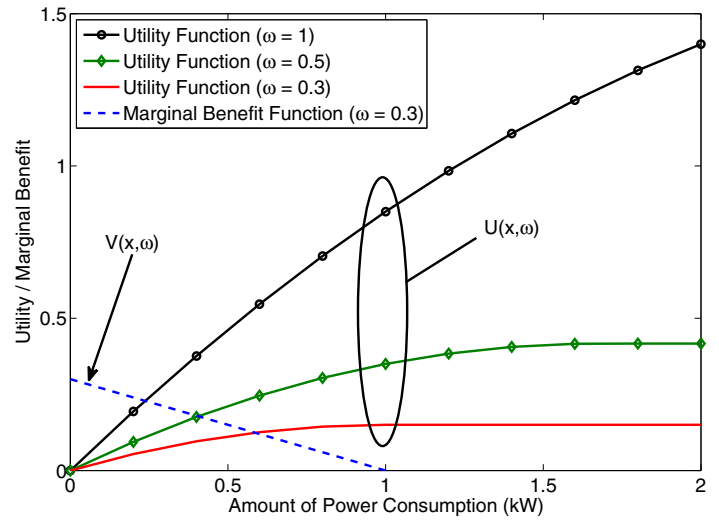

Fig. 1. Sample utility functions for power subscribers $(\alpha=0.3)$.

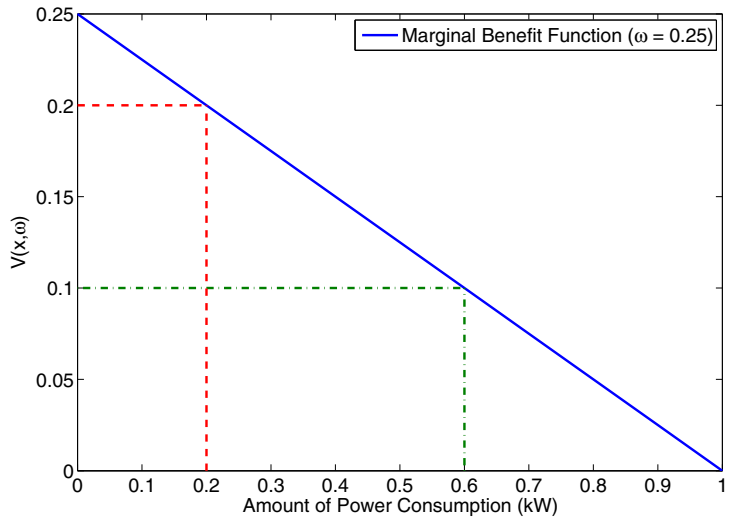

Fig. 2. Different power consumption reactions of a subscriber to two different announced prices $\left(P_{1}=0.2, P_{2}=0.1\right.$ and $\left.\alpha=0.25\right)$.

where $a_{k}>0$ and $b_{k}, c_{k} \geq 0$ are pre-determined parameters.

\section{ReAL-Time Pricing Formulation}

In this section, we formulate the interactions between the users and the energy provider as an optimization problem and analyze the existence and uniqueness of the solution. In our model, the energy provider announces the price of electricity in real-time based on the total load demand.

\section{A. Optimization Problem Formulation}

From a social fairness point of view, it is desirable to utilize the available capacity provided by the energy provider in such a way that the sum of the utility functions of all subscribers is maximized and the cost imposed to the energy provider is minimized. However, each subscriber will choose its consumption level to maximize its own welfare function introduced in (9). These individually optimal consumption levels may not be socially optimal for a general price announced by the energy provider. To align these individual optimal consumption levels with the social optimal case, we need to adopt the sum of all utility functions minus the cost imposed to the energy provider as the objective function while the consumption levels of all users are coupled via the limited available generation capacity. Having a centralized control over all subscribers, and also being provided with complete information about the 
subscribers' needs, an efficient energy consumption schedule can be characterized as the solution of the following problem:

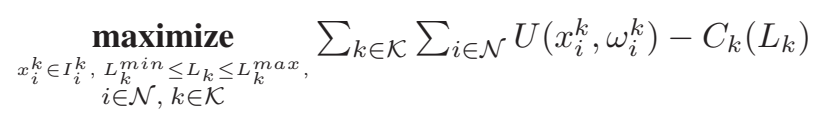$$
\text { subject to } \quad \sum_{i \in \mathcal{N}} x_{i}^{k} \leq L_{k}, \quad \forall k \in \mathcal{K},
$$

where $U\left(x_{i}^{k}, \omega_{i}^{k}\right)$ is defined in (8), $C_{k}\left(L_{k}\right)$ is defined in (12), and $\omega_{i}^{k}$ is the $\omega$ parameter of user $i$ in time slot $k$.

The problem formulated in (13) is a concave maximization problem and can be solved using convex programming techniques such as the interior point method (IPM) [20] in a central fashion. However, the problem arising in solving (13) in a central manner is that we need to know the exact utility function of users. Since it is assumed that the utility parameter $\omega_{i}^{k}$ for each user $i \in \mathcal{N}$ is private, the energy provider may not have sufficient information to solve problem (13).

\section{B. Dual Decomposition Approach}

We notice that (13) can be solved independently for each time slot $k \in \mathcal{K}$. In other words, we have the following optimization problem for each fixed time slot $k \in \mathcal{K}$ :

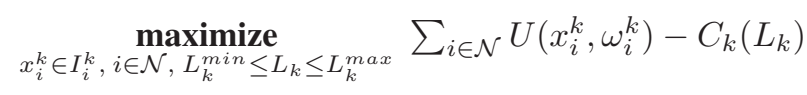

$$
\text { subject to } \quad \sum_{i \in \mathcal{N}} x_{i}^{k} \leq L_{k} \text {. }
$$

Problem (14) is again convex and can be solved easily in a centralized manner. In practice, this problem has to be solved in a distributed fashion. Although the objective function in (14) is further separable in $x_{i}^{k}$ and $L_{k}$, the variables $x_{i}^{k}$ and $L_{k}$ are coupled by the imposed constraint that the total consumed power cannot exceed the available capacity in (14).

For primal problem (14), the Lagrangian is defined as [20]:

$$
\begin{aligned}
\mathcal{L}\left(\mathbf{x}, L_{k}, \lambda^{k}\right)= & \sum_{i \in \mathcal{N}} U\left(x_{i}^{k}, \omega_{i}^{k}\right)-C_{k}\left(L_{k}\right) \\
& -\lambda^{k}\left(\sum_{i \in \mathcal{N}} x_{i}^{k}-L_{k}\right), \\
= & \sum_{i \in \mathcal{N}}\left(U\left(x_{i}^{k}, \omega_{i}^{k}\right)-\lambda^{k} x_{i}^{k}\right) \\
& +\lambda^{k} L_{k}-C_{k}\left(L_{k}\right),
\end{aligned}
$$

where $\lambda^{k}$ is the Lagrange multiplier and $\mathbf{x}=\left(x_{i}^{k}, i \in \mathcal{N}\right)$ for a fixed $k \in \mathcal{K}$. Due to the separability of the first term in the Lagrangian, we can write the objective function of the dual optimization problem as [20]:

$$
\begin{aligned}
\mathcal{D}\left(\lambda^{k}\right) & =\underset{x_{i}^{k} \in I_{i}^{k}, i \in \mathcal{N}, L_{k}^{\min } \leq L_{k} \leq L_{k}^{\max }}{\operatorname{maximize}} \mathcal{L}\left(\mathbf{x}, L_{k}, \lambda^{k}\right) \\
& =\sum_{i \in \mathcal{N}} B_{i}^{k}\left(\lambda^{k}\right)+S_{k}\left(\lambda^{k}\right),
\end{aligned}
$$

where

$$
B_{i}^{k}\left(\lambda^{k}\right)=\underset{x_{i}^{k} \in I_{i}^{k}}{\operatorname{maximize}} U\left(x_{i}^{k}, \omega_{i}^{k}\right)-\lambda^{k} x_{i}^{k},
$$

and

$$
S_{k}\left(\lambda^{k}\right)=\underset{L_{k}^{\text {min }} \leq L_{k} \leq L_{k}^{\max }}{\operatorname{maximize}} \lambda^{k} L_{k}-C_{k}\left(L_{k}\right)
$$

The dual problem is

$$
\underset{\lambda^{k}>0}{\operatorname{minimize}} \mathcal{D}\left(\lambda^{k}\right) .
$$

The first term in $\mathcal{D}\left(\lambda^{k}\right)$ in (16) can be decomposed into $N$ separable subproblems in form of (17), which can be solved by the users, and another subproblem in form of (18), which can be solved by the energy provider.

We can show that strong duality holds, and we can solve the dual problem (19) instead of the primal problem (14). In this case, we can obtain the solution of the dual problem $\lambda^{k *}$, and each individual subscriber and also the energy provider can simply solve their own local optimization problem determined by (17) and (18) to obtain $x_{i}^{k *}$ and $L_{k}^{*}$, respectively.

The key idea which motivates us to propose a real-time pricing algorithm can be understood if we compare the local problem (17) that has to be solved by each individual user with (9), introducing each user's welfare. In fact, if the energy provider would be able to charge the users at a rate $P=\lambda^{k *}$, and each individual user tries to maximize its own welfare function, it will be guaranteed by strong duality that the total power consumption will not exceed the provided capacity.

\section{Distributed Algorithm}

We explained in the previous section that by charging the users with the solution of the dual problem $\lambda^{k *}$, we can achieve the solution of primal problem (14). Interestingly, it is possible to solve the dual problem in an iterative manner using the gradient projection method, and in this case we have

$$
\begin{aligned}
\lambda_{t+1}^{k} & =\left[\lambda_{t}^{k}-\gamma \frac{\partial \mathcal{D}\left(\lambda_{t}^{k}\right)}{\partial \lambda^{k}}\right]^{+} \\
& =\left[\lambda_{t}^{k}+\gamma\left(\sum_{i \in \mathcal{N}} x_{i}^{k *}\left(\lambda_{t}^{k}\right)-L_{k}^{*}\left(\lambda_{t}^{k}\right)\right)\right]^{+},
\end{aligned}
$$

where $t \in \mathcal{T}$, and $\mathcal{T}$ is the set of time instances at which the energy provider updates $\lambda^{k}$. Here, $x_{i}^{k *}\left(\lambda_{t}^{k}\right)$ is the local optimizer of (17), and $L_{k}^{*}\left(\lambda_{t}^{k}\right)$ is the local optimizer of (18) for a given $\lambda_{t}^{k}$, respectively. Also, $\lambda_{t}^{k}$ is the value of $\lambda^{k}$ in instance $t \in \mathcal{T}$, and $\gamma$ is the step size. The interaction between the energy provider and the subscribers is depicted in Fig. 3.

The distributed algorithms of each subscriber and the energy provider are summarized in Algorithms 1 and 2, respectively. Consider Algorithm 1. In Line 1, each subscriber starts with its initial condition, which is assumed to be random. Then, the loop in Lines 2 to 6 describes the responses of each subscriber to the newly announced price $\lambda^{k}$. Within this loop, each subscriber receives the new value of $\lambda^{k}$ in Line 3 and solves local problem (17) to get the optimal consumption $x_{i}^{k *}\left(\lambda^{k}\right)$ corresponding to the new value of $\lambda^{k}$ in Line 4 . In Line 5, the user communicates the new value of $x_{i}^{k *}\left(\lambda^{k}\right)$ to the energy provider. We note that in each time slot $k \in \mathcal{K}$, users apply their new loads only after the algorithm has converged.

In Algorithm 2, the energy provider starts with random initial conditions in Line 1. The loop in Lines 2 to 11 continues during the operational cycle of the system. Within this loop, the energy provider updates $\lambda^{k}$ in each instance $t \in \mathcal{T}$ in Lines 4 and 5. It further calculates the new value of $L_{k}\left(\lambda^{k}\right)$ which 


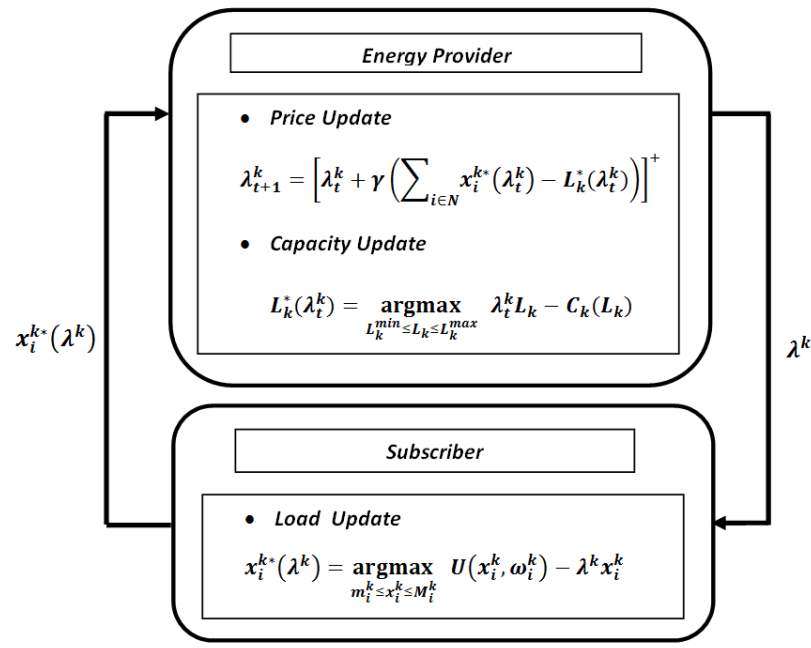

Fig. 3. Illustration of the operation of the proposed algorithm and the interactions between the energy provider and subscribers in the system.

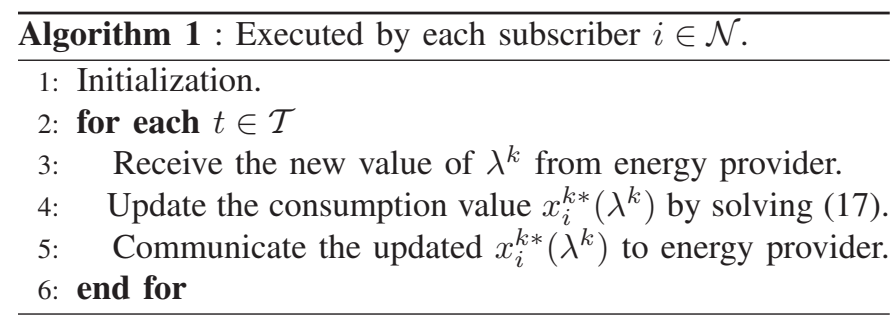

maximizes its welfare and updates its information about the total consumption level of the system in Lines 7 to 9 .

We note that network utility maximization has already been applied successfully in computer networking. The problem formulation in this section is similar to the congestion control problem in the Internet (e.g., [19]). However, the pricing algorithm in this paper differs from the rate allocation problem for the Internet in two aspects: (a) The capacity can be adjusted by the energy provider and may change periodically while the capacity constraint in [19] is fixed; (b) We consider the energy cost imposed to the energy provider and formulate the problem as utility maximization together with cost minimization.

\section{PERFormance EVAluation}

In this section, we present simulation results and assess the performance of our proposed distributed algorithm. In our simulation model, we assume there are $N=10$ subscribers. The entire time cycle is divided into 24 time slots representing the 24 hours of the day. The minimum and the maximum power requirements of all users vary in each time slot, and the minimum generating capacity to meet the minimum power requirements is guaranteed. However, we also assume the maximum generating capacity $L_{k}^{\max }$ is equal to the maximum total power requirements of all the users, so we have $L_{k}^{\max }=\sum_{i \in \mathcal{N}} M_{i}^{k}$, for all $k \in \mathcal{K}$.

We also assume the $\omega$ parameter of each user is selected randomly from the interval $[1,4]$ and remains fixed within the entire cycle. Parameter $\alpha$ of the utility function introduced in

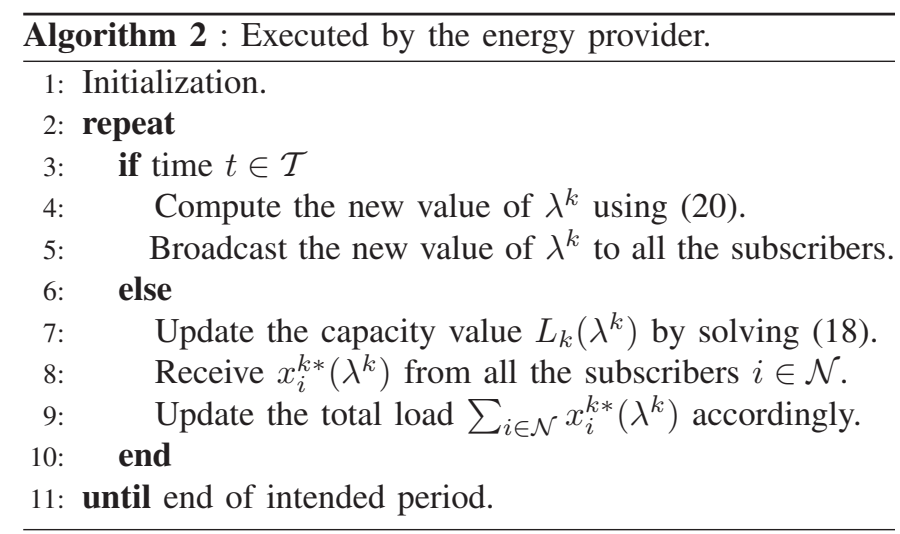

(8) is chosen to be 0.5 , and we set the parameters of the cost function introduced in (12) to $a_{k}=0.01, b_{k}=0$, and $c_{k}=0$.

Simulation results for the total consumed power for the proposed algorithm are shown in Fig. 4. As illustrated in Fig. 4, due to real-time interaction of the subscribers and the energy provider, the two curves corresponding to the total power consumption of the users and the desired generating capacity of the energy provider coincide. The high utilization of the available resources while keeping the total power consumption below the desired threshold is one of the advantages of the proposed algorithm. As expected, the generating capacity and also the total power consumption are bounded within the minimum and the maximum total power requirements of all the users in each time slot.

To have a baseline scheme for comparison with the proposed real-time pricing strategy, we also consider a fixed pricing scenario with a hard constraint to keep the total consumption below the generating capacity without interaction with the users. In the fixed pricing algorithm, the energy provider announces a price for each time slot $k \in \mathcal{K}$ at the beginning of the time slot which guarantees for any type of users with different choices of the $\omega$ parameter that the total consumption level will not exceed the generating capacity. Therefore, in the fixed pricing algorithm, the worst case situation where the $\omega$ parameter of all the users assumes the maximum value $\omega_{\max }=4$ is being considered. Hence, the price in each time slot $k \in \mathcal{K}$ can be calculated as

$$
P_{\text {fixed }}^{k}=\omega_{\max }-\frac{L^{k} \alpha}{N} .
$$

Simulation results for the aggregate utility of all users for the two different methods are shown in Fig. 5. We can see that the aggregate utility is much higher for our proposed real-time pricing algorithm than for the fixed pricing algorithm.

Last but not least, our proposed distributed real-time pricing algorithm can also benefit the users. Let us consider 24 time slots with different power requirements for different users in each time slot. Simulation results for the time averaged welfare of each individual subscriber for our proposed realtime pricing algorithm as well as the fixed pricing algorithm are shown in Fig. 6. We can see that the average welfare of each individual subscriber is much higher for our proposed 


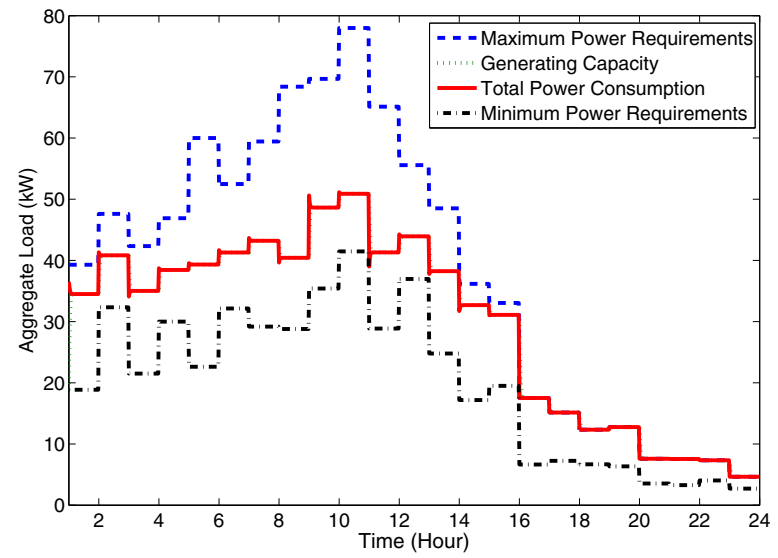

Fig. 4. Total consumed power when the proposed pricing algorithm is used.

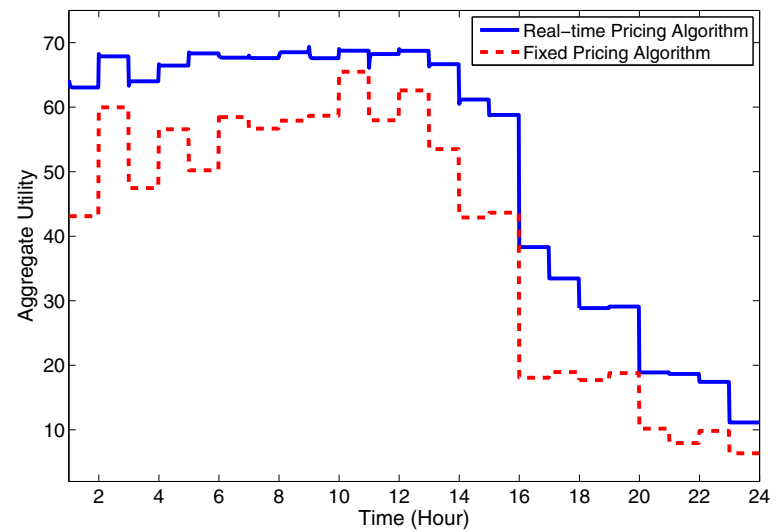

Fig. 5. Obtained aggregate utility of all users when our proposed real-time pricing algorithm as well as a fixed pricing algorithm are used.

algorithm than for the fixed pricing algorithm.

\section{CONCLUSions}

In this paper, we proposed an optimal real-time pricing algorithm for DSM in the future smart grid. The proposed algorithm is based on utility maximization. It can be implemented in a distributed manner to maximize the aggregate utility of all users and minimize the cost imposed to the energy provider while keeping the total power consumption below the generating capacity. Simulation results confirmed that by using our proposed optimization-based real-time pricing model, not only the energy provider, but also the users will benefit. The ideas developed in this paper can be extended in several directions. A system with multiple energy providers can be considered. The effect of malicious users can also be explored.

\section{ACKNOWLEDGMENT}

This research is supported by the Natural Sciences and Engineering Research Council (NSERC) of Canada.

\section{REFERENCES}

[1] L. H. Tsoukalas and R. Gao, "From smart grids to an energy internet: Assumptions, architectures, and requirements," in Proc. of Third Int'l. Conf. on Electric Utility Deregulation and Restructuring and Power Technologies, Nanjing, China, Apr. 2008.

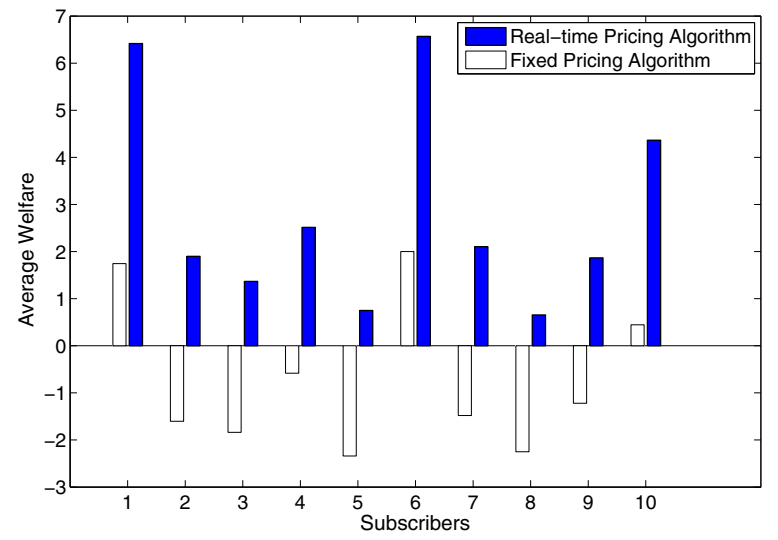

Fig. 6. Average welfare of each subscriber when our proposed real-time pricing algorithm as well as a fixed pricing algorithm are used.

[2] U.S. Department of Energy, The 2008 Buildings Energy Data Book. Energy Efficiency and Renewable Energy, Mar. 2009.

[3] M. Fahrioglu, M. Fern, and F. Alvarado, "Designing cost effective demand management contracts using game theory," in Proc. of IEEE Power Eng. Soc. 1999 Winter Meeting, New York, NY, Jan. 1999.

[4] M. Fahrioglu and F. Alvarado, "Using utility information to calibrate customer demand management behavior models," IEEE Trans. on Power Systems, vol. 16, no. 2, pp. 317-322, May 2001.

[5] R. Faranda, A. Pievatolo, and E. Tironi, "Load shedding: A new proposal," IEEE Trans. on Power Systems, vol. 22, no. 4, pp. 20862093, Nov. 2007.

[6] N. Ruiz, I. Cobelo, and J. Oyarzabal, "A direct load control model for virtual power plant management," IEEE Trans. on Power Systems, vol. 24, no. 2, pp. 959-966, May 2009.

[7] C. Gellings, "The concept of demand-side management for electric utilities," Proceedings of the IEEE, vol. 73, no. 10, pp. 1468-1470, Oct. 1985.

[8] M. Fahrioglu and F. Alvarado, "Designing incentive compatible contracts for effective demand management," IEEE Trans. on Power Systems, vol. 15 , no. 4, pp. 1255-1260, Nov. 2000.

[9] B. Ramanathan and V. Vittal, "A framework for evaluation of advanced direct load control with minimum disruption," IEEE Trans. on Power Systems, vol. 23, no. 4, pp. 1681-1688, Oct. 2008.

[10] A. H. Mohsenian-Rad, V. W. S. Wong, J. Jatskevich, and R. Schober, "Optimal and autonomous incentive-based energy consumption scheduling algorithm for smart grid," in Proc. of IEEE PES Conf. on Innovative Smart Grid Technologies, Gaithersburg, MD, Jan. 2010.

[11] A. H. Mohsenian-Rad and A. Leon-Garcia, "Optimal residential load control with price prediction in real-time electricity pricing environments," IEEE Trans. on Smart Grid, vol. 1, no. 2, Sept. 2010.

[12] P. Luh, Y. Ho, and R. Muralidharan, "Load adaptive pricing: An emerging tool for electric utilities," IEEE Trans. on Automatic Control, vol. 27, no. 2, pp. 320-329, Apr. 1982.

[13] Y. Tang, H. Song, F. Hu, and Y. Zou, "Investigation on TOU pricing principles," in Proc. of IEEE PES Transmission and Distribution Conf. Exhibition: Asia and Pacific, Dalian, China, Aug. 2005.

[14] M. Crew, C. Fernando, and P. Kleindorfer, "The theory of peak-load pricing: A survey," Journal of Regulatory Economics, vol. 8, no. 3, pp. 215-248, Nov. 1995.

[15] S. Zeng, J. Li, and Y. Ren, "Research of time-of-use electricity pricing models in China: A survey," in Proc. of IEEE Int'l. Conf. on Industrial Engineering and Engineering Management, Singapore, Dec. 2008.

[16] U.S. Department of Energy, The Smart Grid: An Introduction, 2009.

[17] A. Vojdani, "Smart integration," IEEE Power and Energy Magazine, vol. 6, no. 6, pp. 72-79, Nov. 2008.

[18] A. Mas-Colell, M. D. Whinston, and J. R. Green, Microeconomic Theory, 1st ed. USA: Oxford University Press, 1995.

[19] S. Low and D. Lapsley, "Optimization flow control I: Basic algorithm and convergence," IEEE/ACM Trans. on Networking, vol. 7, no. 6, pp. 861-874, Dec. 1999.

[20] S. Boyd and L. Vandenberghe, Convex Optimization. Cambridge University Press, 2004. 